\title{
Gestantes colonizadas pelo Streptococcus do grupo B e seus recém- nascidos: análise crítica da conduta adotada no Instituto Fernandes Figueira, Fundação Oswaldo Cruz
}

\author{
Beta-hemolytic streptococcus in pregnant women and their newborn infants: a critical analysis of the
} protocol used at Fernandes Figueira Institute, Oswaldo Cruz Foundation, in Brazil

Natalie Del-Vecchio L. Costa1 ${ }^{1}$ Manoel de Carvalho², Sheila Moura Pone ${ }^{3}$, Saint Clair G. Júnior ${ }^{4}$

\section{RESUMO}

Objetivo: Avaliar a aplicação do protocolo do Centers of Disease Control (CDC, 2002) quanto à profilaxia da sepse neonatal precoce por Streptococcus do grupo B (SGB).

Métodos: Estudo retrospectivo com revisão de prontuários de 125 gestantes colonizadas pelo SGB e 133 recémnascidos, no período de janeiro/2003 a dezembro/2006. A conduta intraparto foi considerada correta quando a gestante recebia antibioticoprofilaxia pelo menos quatro horas antes do parto, ou quando não recebia, mas era submetida a parto cesáreo eletivo. A conduta intraparto foi considerada incorreta quando a gestante recebia antibioticoprofilaxia menos de quatro horas antes do parto, a prescrição antibiótica estava incorreta ou quando não havia profilaxia prescrita.

Resultados: A prevalência de colonização materna pelo SGB foi de 4,7\%. A época de coleta do swab vaginal/retal variou entre 14 e 40 semanas de gestação, com média de 32 semanas. Das gestantes colonizadas, 54 (43\%) receberam conduta intraparto correta. Dos 133 recém-nascidos estudados, $95(71 \%)$ receberam avaliação diagnóstica corretamente, 17 (13\%) evoluíram com sepse clínica e um (0,75\%) apresentou sepse comprovada. A incidência de sepse foi maior em recém-nascidos cujas mães não receberam profilaxia intraparto corretamente, porém esta associação não apresentou diferença estatística significativa (18 versus $7 \%, p>0,05)$.
Conclusões: Apesar de o protocolo para prevenção de sepse precoce pelo SGB estar implementado na instituição, ainda é possível observar falhas na profilaxia intraparto materna. Essas falhas representam oportunidades perdidas na prevenção da sepse precoce pelo SGB.

Palavras-chave: streptococcus agalactiae; antibioticoprofilaxia; sepse; fidelidade a diretrizes.

\section{ABSTRACT}

Objective: To evaluate the use of the guidelines of the Centers of Disease Control (CDC, 2002) regarding the prophylaxes of group B Streptococcus (GBS) early onset neonatal sepsis.

Methods: We conducted a retrospective study by chart review of 125 pregnant women colonized by GBS and 133 neonates born at a $3^{\text {rd }}$ level maternity hospital, from January/2003 to December/2006. The intrapartum management was deemed correct when pregnant women were given prophylactic antibiotic at least four hours before delivery or when they did not receive medication but were submitted to elective cesarean section. The intrapartum management was considered incorrect when the pregnant woman was given antibiotic prophylaxis less than four hours before delivery, when the antibiotic prescription was inadequate or no prophylaxis had been prescribed.
Instituição: Universidade Federal Fluminense (UFF), Rio de Janeiro, RJ, Brasil

${ }^{1}$ Mestre; Tecnologista Júnior do Departamento de Neonatologia do Instituto Fernandes Figueira (IFF) da Fundação Oswaldo Cruz (Fiocruz), Rio de Janeiro, RJ, Brasil

2Doutor; Professor Adjunto da UFF; Neonatologista do IFF da Fiocruz, Rio de Janeiro, RJ, Brasil

${ }^{3}$ Mestre; Tecnologista Pleno II Júnior do Departamento de Doenças Infecciosas em Pediatria do IFF da Fiocruz, Rio de Janeiro, RJ, Brasil

${ }^{4}$ Doutor; Pesquisador em Saúde Pública do Departamento de Neonatologia do IFF da Fiocruz, Rio de Janeiro, RJ, Brasil
Endereço para correspondência: Natalie Del-Vecchio Lages Costa Avenida Rui Barbosa, 716 - Flamengo CEP 22250-020 - Rio de Janeiro/RJ

E-mail: natalie@iff.fiocruz.br

Conflitos de interesse: nada a declarar

Recebido em: 26/6/09

Aprovado em: 30/11/09 
Results: The prevalence of maternal colonization by GBS was $4.7 \%$. The time when the vaginal/rectal swab was collected ranged between 14-40 (mean 32) weeks of gestation. Among the colonized mothers, 54 (43\%) received correct intrapartum management. Among 133 studied infants, 95 (71\%) received a correct diagnosis; $17(13 \%)$ developed clinical sepsis and one $(0.75 \%)$ had proven bacterial sepsis. The incidence of sepsis was higher in infants whose mothers did not receive a correct intrapartum prophylaxis, but this difference was not significant (18 versus $7 \%, p>0.05$ ).

Conclusions: Although the guidelines to prevent perinatal GBS disease are in place, there are flaws in the intrapartum prophylaxis and in infants' evaluation. These flaws represent missed opportunities to prevent early onset GBS sepsis.

Key-words: streptococcus agalactiae; antibiotic prophylaxis; sepsis; guideline adherence.

\section{Introdução}

O Streptococcus agalactiae (SGB) foi reconhecido como causa importante de morbidade e mortalidade neonatal nos Estados Unidos no início de $1970^{(1-4)}$, com taxa de mortalidade de 15 a $50 \%$.

O fator determinante para infecção neonatal pelo SGB é a presença desse micro-organismo no trato genital materno no momento do nascimento. A prevalência de colonização no trato genital em mulheres grávidas varia de 10 a $30 \%{ }^{(5,6)}$ e a transmissão vertical ocorre em 30 a $70 \%$ de neonatos cujas mães são colonizadas pelo SGB na gestação ${ }^{(1)}$.

Recomendações de profilaxia intraparto para prevenir doença perinatal pelo SGB de início precoce foram revisadas recentemente pelo Centers for Disease Control and Prevention $(\mathrm{CDC})^{(7)}$. A incidência norte-americana de doença precoce pelo SGB após a introdução da quimioprofilaxia intraparto declinou de 1,8 casos/1.000 nascidos vivos em 1990 para 0,32 casos/1.000 nascidos vivos em $2003^{(8,9)}$. A quimioprofilaxia intraparto não é rotineiramente recomendada para gestantes submetidas a parto cesáreo eletivo (sem início de trabalho de parto e com membrana amniótica íntegra) $)^{(7)}$.

A epidemiologia da doença causada pelo SGB, tanto em mulheres grávidas quanto em recém-nascidos $(\mathrm{RN})$, tem sido amplamente estudada nos Estados Unidos e na Europa, porém poucos estudos abordaram esse assunto na América Latina. Dados na literatura nacional apontam o SGB como principal agente de sepse neonatal precoce ${ }^{(10)}$. Segundo estu- dos brasileiros recentes ${ }^{(11,12)}$, as prevalências de colonização materna pelo SGB variam de 14,9 a $21,6 \%$.

O Instituto Fernandes Figueira (IFF), unidade da Fundação Oswaldo Cruz (Fiocruz), é um hospital terciário de referência para gestantes e $\mathrm{RN}$ de alto risco, onde a realização da cultura de swab vaginal/retal no pré-natal para detecção da colonização pelo SGB é rotineira. O presente estudo objetivou avaliar a aplicação do protocolo do CDC $2002^{(7)}$ pelas equipes de neonatologia e obstetrícia do IFF em gestantes colonizadas pelo SGB e seus RN.

\section{Métodos}

Trata-se de um estudo de coorte retrospectivo, descritivo, baseado na revisão de prontuários de gestantes colonizadas pelo SGB (swab vaginal/retal positivo) que realizaram prénatal no Instituto Fernandes Figueira no período de $1^{\circ}$ janeiro de 2003 a 31 de dezembro de 2006 e na análise dos prontuários de seus RN.

O serviço de obstetrícia do IFF segue o protocolo do CDC de $2002^{(7)}$ para prevenção de doença perinatal pelo SGB, realizando rotineiramente swab vaginal/retal de todas as gestantes no pré-natal, entre 35 e 37 semanas de gestação. O material para pesquisa do SGB é colhido com um único swab estéril da vagina e reto. Depois da coleta, os swabs são encaminhados em meio de transporte (Cary-Blair) ao laboratório e são semeados em ágar sangue de carneiro. Após 24 horas de incubação, as placas são inspecionadas e as colônias sugestivas de SGB ( $\beta$ hemolíticas ou não, gram-positivas e catalase negativas) são identificadas. Quando o estreptococo $\beta$ hemolítico não é identificado após 18 a 24 horas de incubação em placa de ágar, as placas são reincubadas por mais 24 horas e a leitura final, efetuada em 48 horas. Todas as colônias suspeitas de SGB são submetidas ao teste de aglutinação pelo látex para detecção do antígeno específico do grupo B e ao teste de CAMP (Christie-Atkins-Munch-Petersen). A colonização materna é definida com isolamento do SGB no swab por cultura em meio não-seletivo.

O estudo incluiu todas as gestantes colonizadas pelo SGB no período estudado e seus respectivos RN. Foram excluídos do estudo $\mathrm{RN}$ portadores de malformação congênita e síndrome genética devido às dificuldades encontradas na avaliação diagnóstica. Foram excluídas gestantes que evoluíram para aborto espontâneo ou cuja gestação resultou em óbito fetal.

O projeto de pesquisa foi aprovado pelo Comitê de Ética em Pesquisa em Seres Humanos do Instituto Fernandes Figueira. 
Foi considerada profilaxia presente em todos os casos de gestante colonizada pelo SGB que receberam pelo menos uma dose de antibiótico (ampicilina) no período intraparto. A conduta foi considerada correta quando a gestante recebeu antibioticoprofilaxia intraparto pelo menos quatro horas antes do parto. A conduta também foi considerada correta na gestante que não recebia profilaxia intraparto porque foi submetida a parto cesáreo eletivo com a bolsa amniótica íntegra e sem evidências de trabalho de parto. A conduta foi definida como incorreta quando a antibioticoprofilaxia ocorria há menos de quatro horas do parto, se a prescrição antibiótica estava incorreta ou se não havia profilaxia prescrita. A prescrição médica foi considerada incorreta quando a dose ou o intervalo dos antibióticos não estava de acordo com as recomendações do protocolo do CDC $2002^{(7)}$ ou quando o antibiótico utilizado não era o proposto para profilaxia do SGB.

Os $\mathrm{RN}$ foram avaliados por meio da análise dos prontuários de acordo com a evolução clínica e exames laboratoriais, sendo então classificados como sepse comprovada, sepse clínica e não sepse. Os critérios utilizados para definir síndrome infecciosa foram propostos por Tollner ${ }^{(13)}$ e utilizados por Geme et $a l^{(14)}$, Garner et $a l^{(15)}$, Donowitz ${ }^{(16)}$, Yancey et $a l^{(17)}$, Messer $e t a l^{(18)}$, Panero et $a l^{(19)}$ e Silveira e Procianoy ${ }^{(20)}$. De acordo com esses critérios, para que o $\mathrm{RN}$ seja considerado séptico, é necessária a presença de pelo menos um sinal de três categorias clínicas ou um sinal de duas categorias clínicas associados à presença de um exame laboratorial alterado e/ou um fator de risco materno (Quadro 1). Definiu-se como sepse comprovada quando o RN apresentou síndrome infecciosa e comprovação laboratorial (hemocultura positiva para SGB). Definiu-se sepse clínica quando o neonato mostrou síndrome infecciosa, porém sem crescimento de micro-organismos em culturas. Finalmente, foram considerados $\mathrm{RN}$ sem sepse aqueles que não apresentaram quadro clínico ou laboratorial sugestivo de síndrome infecciosa.

Para avaliação das hemoculturas, utilizou-se o método automatizado BACTEC 912,0 produzido pela Becton Dickinson $L t d a$, realizado no laboratório de bacteriologia do IFF.

De acordo com o algoritmo do $\mathrm{RN}$ do protocolo do CDC $2002^{(7)}$, os seguintes critérios foram adotados para avaliação diagnóstica do $\mathrm{RN}$ : para aqueles com idade gestacional $\geq 35$ semanas cuja mãe recebeu profilaxia correta, nenhuma avaliação ou terapia foi recomendada caso estivesse assintomático. Nesse caso, a recomendação foi observar o $\mathrm{RN}$ por $\geq 48$ horas. Os $\mathrm{RN}$ assintomáticos $\geq 35$ semanas de idade gestacional, cuja mãe não recebeu profilaxia correta foi submetido à avaliação limitada (rastreamento infeccioso com hemograma e
Quadro 1 - Critérios para definição de síndrome infecciosa no recém-nascido

\section{I - Categorias clínicas}

1. Instabilidade térmica apresentada pelo menos 2 vezes num período de 24 horas

2. Apneia, bradipneia, gemência, taquipneia, retração esternal e subcostais, batimentos de asas de nariz, cianose, aumento da necessidade de oxigênio e dos parâmetros do respirador em recém-nascidos ventilados e previamente estáveis

3. Hipotonia e convulsões

4. Irritabilidade e letargia

5. Sintomas gastrintestinais como distensão abdominal, vômitos, resíduo gástrico e dificuldade de aceitação alimentar

6. Icterícia idiopática

7. Palidez cutânea, pele fria, sudoreica, hipotensão, tempo de enchimento capilar superior a 3 segundos

8. Sinais de sangramento, com quadro clínico sugestivo de coagulação intravascular disseminada

9. Avaliação subjetiva: "RN que parece não estar bem"

II - Fatores de risco materno

1. Febre materna

2. Infecção do trato urinário suspeita ou comprovada, exceto nos casos resolvidos antes do início do trabalho de parto

3. Infecção do trato genital, corioamnionite, líquido amniótico fétido, leucorreia, herpes genital, papiloma vírus e hipotonia uterina

\section{III - Sinais laboratoriais}

1. Leucocitose (acima de $20.000 / \mathrm{mm}^{3}$ )

2. Leucopenia (abaixo de $5.000 / \mathrm{mm}^{3}$ )

3. Relação de neutrófilos imaturos/totais $\geq 0,2$

4. Plaquetopenia $\left(\leq 100.000 / \mathrm{mm}^{3}\right)$

5. Hiperglicemia (>140mg\%)

6. Acidose metabólica inexplicada (BE $\leq 10)$

$\mathrm{RN}$ : recém-nascido; $\mathrm{BE}$ : excesso de bases.

hemocultura) e observado por $\geq 48$ horas. Nos casos de RN assintomáticos $<35$ semanas de idade gestacional, independente de a mãe ter recebido profilaxia correta ou não, o paciente foi avaliado (avaliação limitada com hemograma e hemocultura) e observado por pelo menos 48 horas. Qualquer $\mathrm{RN}$ que tenha evoluído com sinais clínicos de sepse deveria receber avaliação diagnóstica completa, iniciando-se terapia antibiótica. Dessa forma, foi considerada como avaliação correta do RN o uso do algoritmo do CDC $2002^{(7)}$. 
Foi elaborado um banco de dados no programa Epi-Info 3.3, utilizando-se o programa de estatística SPSS versão 13.0 para análise de dados. O teste de qui-quadrado e o teste exato de Fisher foram usados para comparar variáveis categórica e o teste $t$ para comparar variáveis contínuas, sendo significante $p<0,05$.

\section{Resultados}

Durante o período analisado, foram matriculadas 4.075 gestantes no pré-natal no IFF. Dessas, 3.929 gestantes colheram swab vaginal/retal, sendo evidenciada a colonização por SGB em 186 gestantes (4,7\% de colonização materna). Os prontuários dessas 186 gestantes com swabs positivos para SGB foram analisados. Desses, 61 foram excluídos da avaliação (35 partos não ocorreram no IFF, dez prontuários de gestantes não foram encontrados, um prontuário de RN não foi encontrado, quatro óbitos fetais, quatro abortos e sete $\mathrm{RN}$ excluídos devido à malformação congênita). Desse modo, foram revisados prontuários de 125 gestantes colonizadas pelo SGB e $133 \mathrm{RN}$, sendo seis pares de gemelares e um trigemelar.

A idade das gestantes variou entre 14 e 43 anos, sendo a idade média $27 \pm 8$ anos e a mediana, 27 anos. Vinte e nove gestantes

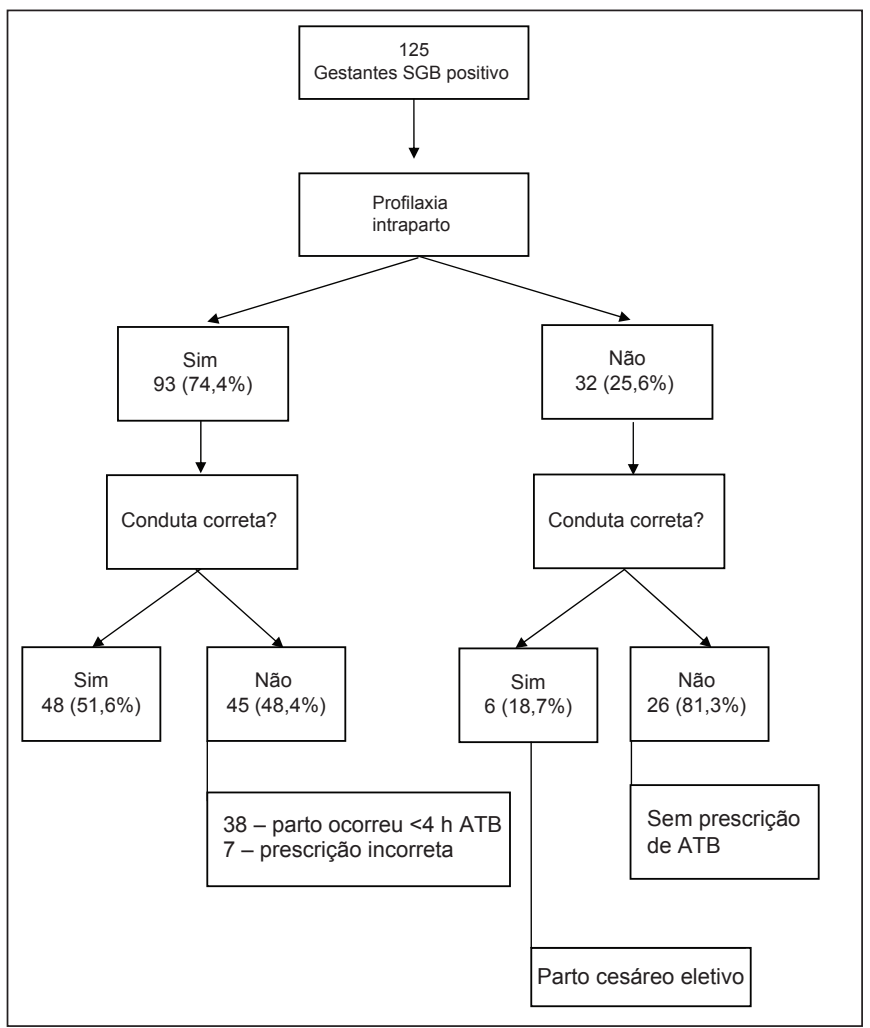

Figura 1 - Profilaxia antibiótica intraparto e conduta materna.
(23\%) eram menores de 20 anos e $22 \%$ apresentavam idade $\geq 35$ anos. Em relação à cor, $65(52 \%)$ gestantes eram brancas. Oitenta e nove (71\%) gestantes eram multíparas. A idade gestacional das pacientes na época da coleta dos swabs variou entre 14 e 40 semanas, com média de 32 e mediana de 34 semanas. Durante o pré-natal, 59 (47\%) gestantes receberam ampicilina via oral no momento em que foi diagnosticada a colonização pelo SGB no swab vaginal/retal. O parto cesáreo ocorreu em 71 (57\%) gestantes. Dezoito gestantes (14\%) apresentaram ruptura de bolsa amniótica com intervalo de tempo entre a ruptura e o parto $\geq 18$ horas e cinco (4\%) gestantes apresentaram febre no momento do parto.

Das 125 gestantes estudadas, $93(74 \%)$ receberam profilaxia intraparto e 32 (26\%) não receberam. Entretanto, das 93 gestantes que receberam profilaxia intraparto, a conduta foi considerada correta em 48 (52\%) e incorreta em 45 (48\%). A conduta foi considerada incorreta porque a profilaxia, mesmo prescrita, não ocorreu como recomendada pelo CDC. Sete gestantes apresentavam prescrição incorreta (dose de ataque incorreta, intervalo entre as doses inadequado) e, em 38 gestantes, mesmo com a profilaxia prescrita, o parto ocorreu em menos de quatro horas após a administração do antibiótico (Figura 1). Das 32 gestantes (26\%) que não receberam profilaxia intraparto, seis (19\%) receberam conduta correta, pois foram submetidas a parto cesáreo eletivo. As outras 26 gestantes (81\%) não apresentavam prescrição de profilaxia antibiótica.

Dos 133 RN estudados, havia seis pares de gêmeos e um trigemelar. A idade gestacional variou entre 28 e 42 semanas, sendo a média $37 \pm 3$ semanas. Quarenta (30\%) $\mathrm{RN}$ eram prematuros, $88(66 \%)$ a termo e cinco (4\%) póstermo. Desses RN, 17 (13\%) evoluíram com sepse clínica e apenas um apresentou sepse comprovada com hemocultura positiva para SGB. Dos RN que desenvolveram sepse, $78 \%$ eram prematuros, em contraste com $22 \%$ de $\mathrm{RN}$ a termo $(p<0,001)$. Não houve caso de sepse comprovada por outro agente infeccioso. A idade gestacional média foi $34 \pm 3$ semanas para os $\mathrm{RN}$ que evoluíram para sepse clínica/comprovada e $38 \pm 2$ semanas para os $\mathrm{RN}$ sem sepse $(p<0,05)$. Dados comparativos dos $\mathrm{RN}$ segundo presença ou ausência de sepse são apresentados na Tabela 1 .

Dos 133 pacientes estudados, a conduta adotada pelo neonatologista foi correta em 95 (71\%) e incorreta em 38 (29\%) casos: dez $\mathrm{RN}$ receberam rastreamento desnecessário, 21 não foram rastreados quando deveriam ter sido e sete foram rastreados sem coleta de hemocultura. Todos os $\mathrm{RN}$ que apresentaram sepse clínica e sepse comprovada receberam avaliação diagnóstica correta pelos neonatologistas. Os $\mathrm{RN}$ 
Tabela 1 - Características dos recém-nascidos segundo presença de sepse clínica e comprovada

\begin{tabular}{|c|c|c|}
\hline & $\begin{array}{c}\text { Sepse neonatal clínica e } \\
\text { comprovada } \\
(n=18)\end{array}$ & $\begin{array}{c}\text { Sepse neonatal ausente } \\
\qquad(n=115)\end{array}$ \\
\hline Idade gestacional (semanas) & $34 \pm 3,4$ & $38 \pm 2,3^{*}$ \\
\hline Peso nascimento $(\mathrm{g})$ & $2121 \pm 939$ & $2952 \pm 680^{*}$ \\
\hline Sexo masculino & 7 & 54 \\
\hline Sexo feminino & 11 & 61 \\
\hline Adequado para idade gestacional & 12 & 84 \\
\hline Grande para idade gestacional & 1 & 5 \\
\hline Pequeno para idade gestacional & 5 & 26 \\
\hline Apgar $1^{\circ}$ minuto (mediana) & 8 & 8 \\
\hline Apgar $5^{\circ}$ minuto (mediana) & 9 & 9 \\
\hline RN em oxigenioterapia & 14 & $10^{*}$ \\
\hline RN em ventilação mecânica & 5 & $2^{*}$ \\
\hline
\end{tabular}

${ }^{*} p<0,05$.

classificados como não-sepse e que não receberam avaliação correta evoluíram bem.

Quando se avaliou a conduta intraparto adotada pelo obstetra e a evolução clínica do RN, observou-se que, das 54 mães nas quais conduta foi correta, apenas quatro (7\%) RN evoluíram para sepse clínica. Em contraste, 13 (18\%) RN das 71 mães que receberam conduta incorreta evoluíram para sepse clínica e um $(1,4 \%)$ evoluiu com sepse comprovada. Apesar de a incidência de sepse clínica e comprovada ter sido maior em RN cujas mães não receberam conduta correta no período intraparto, esta diferença não foi significativa (18 versus $7 \%, p>0,05)$. A conduta intraparto adotada pelos obstetras em 125 gestantes estudadas e a evolução clínica de seus RN são mostradas na Figura 2.

Das quatro gestantes que receberam conduta intraparto correta e cujos RN evoluíram para sepse clínica, três apresentavam bolsa amniótica rota por mais de 18 horas, incluindo uma gestante com febre e leucocitose. Dessas três gestantes, duas apresentaram diagnóstico histopatológico de corioamnionite.

O RN que apresentou sepse comprovada por SGB (hemocultura positiva) era prematuro, com idade gestacional de 34 semanas e evoluiu com síndrome infecciosa nas primeiras horas de vida e exames laboratoriais alterados (leucopenia no primeiro hemograma colhido no momento da internação e proteína $C$ reativa aumentada). Sua mãe era primípara, jovem e apresentava ruptura prolongada de membrana ovular (61 horas).

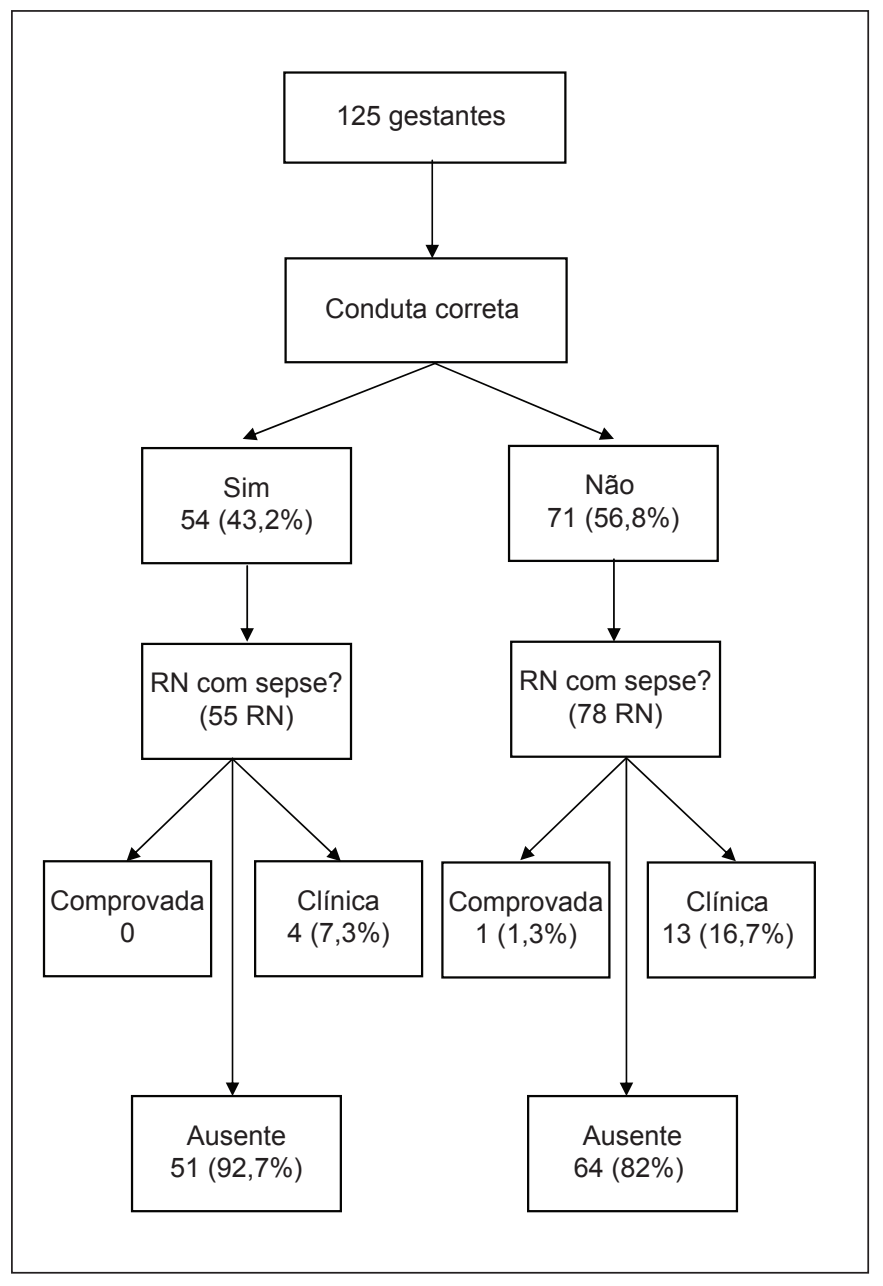

Figura 2 - Profilaxia antibiótica materna intraparto e evolução clínica neonatal. 
Dos $18 \mathrm{RN}$ que evoluíram para sepse, um evoluiu a óbito com seis horas de vida (mãe sem profilaxia intraparto).

\section{Discussão}

O primeiro estudo no Brasil, em 1982(21), detectou uma taxa de colonização materna por SGB de $26 \%$, semelhante à descrita em outros países. Em 1995, em Londrina, Mocelin $e$ t al ${ }^{(22)}$ detectaram taxa de colonização materna de $15 \%$. Entretanto, os sítios pesquisados no estudo foram o vaginal e periuretral e o meio seletivo não foi utilizado. Muitos estudos já demonstraram maior acurácia do método baseado em cultura, com identificação das mulheres colonizadas no momento do parto, para prevenção da sepse neonatal pelo $\mathrm{SGB}^{(23,24)}$. Entretanto, para esse método poder alcançar eficácia máxima, alguns cuidados devem ser observados: sítios anatômicos para coleta da amostra, cuidados no transporte, preservação do material e métodos microbiológicos precisos para cultura e detecção do SGB.

No presente estudo, a taxa de colonização materna pelo SGB foi de $4,7 \%$, prevalência baixa quando comparada à taxa de $10-30 \%$ descrita na literatura internacional ${ }^{(5)}$. A taxa de colonização do presente estudo pode estar subestimada por dois fatores: técnicas microbiológicas empregadas e momento de coleta do swab no pré-natal. O laboratório de bacteriologia do IFF não utiliza o meio seletivo Todd Hewitt para cultura do SGB e os swabs maternos colhidos são semeados diretamente em ágar-sangue de carneiro, cuja sensibilidade é de cerca de $60 \%$, em contraste com $85 \%$ do meio seletivo ${ }^{(25)}$.

Outro fator importante para a positividade da cultura é o momento de coleta no pré-natal. O valor preditivo da cultura no pré-natal é elevado se realizado com 35 semanas de gestação, chegando a quase $100 \%{ }^{(26)}$. Não se sabe em que momento foi colhido o swab das gestantes que não estavam colonizadas, mas, pela característica da clientela do IFF, pode-se inferir que a maioria dos swabs foi colhida antes do momento ideal, fato que pode subestimar a prevalência de colonização em nossa amostra.

Avaliando a conduta obstétrica intraparto, a maioria das gestantes colonizadas não recebeu conduta intraparto correta. Puopolo et a ${ }^{(27)}$ realizaram um estudo em Boston (Harvard Medical School) com o objetivo de avaliar se fatores clínicos, microbiológicos ou de procedimentos poderiam estar contribuindo para a persistência da doença. Seus resultados mostraram que mais da metade das mães dos $\mathrm{RN}$ a termo que não eram colonizadas pelo SGB, mas apresentavam fatores de risco para infecção neonatal, não recebeu antibiótico intraparto. Erros de procedimento foram identificados em quatro casos. Os autores concluem que os casos de sepse precoce pelo SGB continuam a ocorrer e podem ser facilitados por vários aspectos da prática clínica pré-natal ou perinatal, incluindo a acurácia na detecção do SGB, a comunicação dos resultados do rastreamento, a administração da profilaxia intraparto ou a identificação e tratamento dos $\mathrm{RN}$ em risco.

A maioria dos casos de sepse ocorreu nos $\mathrm{RN}$ cujas mães não receberam conduta intraparto corretamente. Foram detectados fatores de risco para infecção em três $\mathrm{RN}$ de quatro mães com conduta obstétrica correta e sepse clínica neonatal. Outro fator que pode ter exercido influência é que, dos quatro RN que evoluíram para sepse clínica, três eram prematuros. A prematuridade é um fator de risco independente para a sepse neonatal. Boyer $e$ al $^{(28)}$ identificaram três situações que aumentam a chance de doença neonatal pelo SGB quando a mãe é colonizada: (1) ruptura prolongada de membrana ovular maior ou igual a 18 horas (risco aumenta sete vezes), (2) febre materna (risco aumenta quatro vezes) e (3) prematuridade (risco aumenta sete vezes). Assim, os RN das mães que receberam conduta correta mas, ainda assim, tiveram sepse clínica apresentavam outros fatores de risco para a sepse neonatal.

Um dado preocupante é o fato de que alguns RN não receberam avaliação correta. A bacteremia pelo SGB pode ocorrer com sinais sistêmicos mínimos ou sem sinais sistêmicos ou focais ${ }^{(29)}$. Diversos estudos têm demonstrado que 4 a $20 \%$ dos $\mathrm{RN}$ a termo com infecção precoce pelo SGB apresentam bacteremia assintomática ${ }^{(30)}$. Assim, o momento ideal da terapia pode ser perdido se não for avaliado corretamente. A realização de hemoculturas de $\mathrm{RN}$ de mães com fatores de risco que não receberam profilaxia intraparto corretamente auxilia a identificar os $\mathrm{RN}$ com bacteremia assintomática e risco de desenvolver sepse de início tardio.

Deve-se considerar que o estudo retrospectivo apresenta limitações, o que pode gerar viés de informação devido a dados incompletos ou incorretos no prontuário. A perda ocorrida no estudo pode ter contribuído para que algumas análises não atingissem significância estatística.

O IFF adota como rotina obstétrica e neonatal o protocolo de prevenção de doença perinatal pelo SGB do CDC $2002^{(7)}$, entretanto, os resultados deste estudo mostraram que a maioria das gestantes colonizadas não recebeu conduta intraparto correta. Esses dados sugerem a necessidade de que cada serviço faça uma análise crítica da conduta adotada na prevenção e tratamento da doença neonatal precoce pelo SGB. 


\section{Referências bibliográficas}

1. Baker CJ, Barrett FF, Gordon RC, Yow MD. Suppurative meningitis due to streptococci of Lancefield group B: a study of 33 infants. J Pediatr 1973;82:724-9.

2. Barton LL, Feigin RD, Lins R. Group B beta hemolytic streptococcal meningitis in infants. J Pediatr 1973;82:719-23.

3. Franciosi RA, Knostman JD, Zimmerman RA. Group B streptococcal neonatal and infant infections. J Pediatr 1973;82:707-18.

4. McCracken GH Jr. Group B streptococci: the new challenge in neonatal infections. J Pediatr 1973;82:703-6.

5. Regan JA, Klebanoff MA, Nugent RP. Vaginal infections and prematurity study group. The epidemiology of group B streptococcal colonization in pregnancy. Obstet Gynecol 1991;77:604-10.

6. Anthony BF, Eisenstadt R, Carter J, Kim KS, Hobel CJ. Genital and intestinal carriage of group $B$ streptococci during pregnancy. $J$ Infect Dis 1981;143:761-6

7. Schrag S, Gorwitz R, Fultz-Butts K, Schuchat A. Prevention of perinatal group $B$ streptococcal disease. Revised guidelines from CDC. MMWR Recomm Rep 2002;51:1-22.

8. Centers for Disease Control and Prevention (CDC). Diminishing racial disparities in early-onset neonatal group B streptococcal disease - United States, 2000-2003. MMWR Morb Mortal Wkly Rep 2004;53:502-5.

9. Zangwill KM, Schuchat A, Wenger JD. Group B streptococcal disease in the United States, 1990: report from a multistate active surveillance system. MMWR CDC Surveill Summ 1992:41:25-32.

10. Vaciloto E, Richtmann R, Costa HP, Kusano EJ, Almeida MF, Amaro ER. A survey of the incidence of neonatal sepsis by group $B$ Streptococcus during a decade in a Brazilian maternity hospital. Braz J Infect Dis 2002;6:55-62.

11. Beraldo C, Brito AS, Saridakis HO, Matsuo T. Prevalence of vaginal and anorectal colonization by group $B$ streptococcus in pregnant women in the last three months of gestation. Rev Bras Ginecol Obstet 2004;26:543-9.

12. Pogere A, Zoccoli CM, Tobouti NR, Freitas PF, d'Acampora AJ, Zunino JN. Prevalência de colonização pelo estreptococo do grupo $B$ em gestantes atendidas em ambulatório de pré-natal. Rev Bras Ginecol Obstet 2005;27:174-80.

13. Töllner U. Early diagnosis of septicemia in the newborn. Clinical studies and sepsis score. Eur J Pediatr 1982;138:331-7.

14. St Geme JW Jr, Murray DL, Carter J, Hobel CJ, Leake RD, Anthony BF et al. Perinatal bacterial infection after prolonged rupture of amniotic membranes: an analysis of risk and management. J Pediatr 1984;104:608-13.

15. Garner JS, Jarvis WR, Emori TG, Horan TC, Hughes JM. CDC definitions for nosocomial infections, 1988. Am J Infect Control 1988;16:128-40.
16. Donowitz LG. Nosocomial infection in neonatal intensive care units. Am J Infect Control 1989;17:250-7.

17. Yancey MK, Duff $P$, Kubilis $P$, Clark $P$, Frentzen $B H$. Risk factors for neonatal sepsis. Obstet Gynecol 1996;87:188-94.

18. Messer J, Eyer D, Donato L, Gallati H, Matis J, Simeoni U. Evaluation of interleukin- 6 and soluble receptors of tumor necrosis factor for early diagnosis of neonatal infection. J Pediatr 1996;129:574-80.

19. Panero A, Pacifico L, Rossi N, Mancuso G, Stegagno M, Chiesa C. Interleukin 6 in neonates with early and late onset infection. Pediatr Infect Dis 1997;16:370-5

20. Silveira RC, Procianoy RS. Evaluation of interleukin-6, tumour necrosis factoralpha and interleukin- 1 beta for early diagnosis of neonatal sepsis. Acta Paediatr 1999;88:647-50

21. Benchetrit LC, Fracalanzza SE, Peregrino H, Camelo AA, Sanches LA. Carriage of Streptococcus agalactiae in women and neonates and distribution of serological types: a study in Brazil. J Clin Microbiol 1982;15:787-90.

22. Mocelin CO, Carvalho DA, Brites C, Christofolli D, Mocelin AO, Fracalanza $\mathrm{SE}$ et al. Isolamento de Streptococcus agalactiae de gestantes na região Londrina-PR. Rev Bras Ginecol Obstet 1995;17:915-8.

23. Schrag SJ, Zell ER, Lynfield R, Roome A, Arnold KE, Craig AS et al. A population-based comparison of strategies to prevent early-onset group $B$ streptococcal disease in neonates. N Engl J Med 2002;347:233-9.

24. Apgar BS, Greenberg G, Yen G. Prevention of group B streptococcal disease in the newborn. Am Fam Physician 2005;71:903-10.

25. McKenna DS, lams JD. Group B streptococcus infections. Semin Perinato 1998;22:267-76

26. Gotoff SP, Boyer KM. Prevention of early-onset neonatal group B streptococca disease. Pediatrics 1997;99:866-9.

27. Puopolo KM, Madoff LC, Eichenwald EC. Early-onset group B streptococcal disease in the era of maternal screening. Pediatrics 2005;115:1240-6.

28. Boyer KM, Gadzala CA, Kelly PD, Burd LI, Gotoff SP. Selective intrapartum chemoprophylaxis of neonatal group B streptococcal early-onset disease. II. Predictive value of prenatal cultures. J Infect Dis 1983;148:802-9.

29. Baker CJ, Edwards MS, Nizet V. Group B streptococcal infections. In Remington JS, Klein JO, editors. Infectious diseases of the fetus and newborn infant. $6^{\text {th }}$ ed. Philadelphia: Elsevier Saunders; 2006. p. 403-64.

30. Bromberger P, Lawrence JM, Braun D, Saunders B, Contreras R, Petitti DB. The influence of intrapartum antibiotics on the clinical spectrum of earlyonset group B streptococcal infection in term infants. Pediatrics 2000;106: $244-50$ 